\title{
INCIDÊNCIA DE LESÕES EM PRATICANTES DE CORRIDA DE RUA NO MUNICÍPIO DE CRICIÚMA, BRASIL
}

\author{
INCIDENCE OF INJURIES ON STREET RUNNING PRACTITIONERS IN CRICIÚMA CITY, BRAZIL
}

\author{
INCIDENCIA DE LESIONES EN LOS PRACTICANTES DE CARRERAS DE CALLE EN EL MUNICIPIO DE \\ CRICIÚMA, BRASIL
}

Gabriel Mamoru Masuda Rangel' (Educador Físico).

Joni Márcio de Farias²

(Educador Físico)

1. Formado em Educação Física na Universidade do Extremo Sul Catarinense (UNESC), Criciúma, SC, Brasil.

2. Professor do Programa de Mestrado em Saúde Coletiva e Coord. Grupo de Estudos e Pesquisa em Promoção da Saúde da Universidade do Extremo Sul Catarinense (UNESC), Criciúma, SC, Brasil.

\section{Correspondência:}

Rua: José Piazza, 200, Jardim Maristela, Criciúma, SC, Brasil. 88815-280. gabrielmamoru@hotmail.com

\section{RESUMO}

Introdução: Observa-se um pronunciado aumento da popularidade das corridas de rua; consequentemente, há mais adeptos dessa prática. Assim sendo, vários estudos vêm buscando diversas abordagens da modalidade, visando desvendar os mecanismos que a compõem. Objetivo:Verificar a incidência de lesões em praticantes de corrida de rua do município de Criciúma, SC, assim como outros fatores associados, como perfil dos praticantes, características do treinamento e existência de acompanhamento profissional. Métodos: Foi realizado um estudo descritivo transversal com 88 corredores ( 56 homens e 32 mulheres). Resultados: A média de idade foi 36 anos, sendo que a maioria dos participantes praticava corrida três vezes por semana $(55,4 \%)$ e, do total da amostra, 43,2\% já haviam tido alguma lesão, sendo o joelho (52,6\%) o local mais acometido. Entre os que já tinham sofrido lesão, houve forte correlação entre quantidade de lesões e o tempo de prática da modalidade $(r=0,269)$ e os que percorriam maior distância média diária de treino $(r=0,226)$. No entanto, os dados demonstraram que o trabalho preventivo não foi eficaz na diminuição de incidência de lesão $(r=-0,133)$. Conclusão: Há uma relação direta entre distância percorrida e lesão, bem como o tempo de prática. A orientação de profissionais não tem influência significativa na redução das lesões, ou seja, muitas lesões podem ser decorrentes do volume e da intensidade dos treinos prescritos ou executados de maneira equivocada, não respeitando o condicionamento físico atual e a individualidade biológica, na perspectiva de superar seus próprios limites de qualquer modo. Ressaltamos a importância de mais estudos que relacionem a progressão de treinamento, lesão e prevenção.

Descritores: corrida; ferimentos e lesões; educação física e treinamento.

\section{ABSTRACT}

Introduction: There has been a marked increase in the popularity of street running; consequently, more people are taking up this sport. Therefore, studies are seeking different approaches to this modality, aiming to unravel the mechanisms that compose it. Objective: To verify the incidence of injuries in street runners of Criciúma city, SC, as well as other related factors such as profile of practitioners, training characteristics, and the existence of professional monitoring. Methods: A cross-sectional descriptive study with 88 runners (56 men and 32 women) was conducted. Results: The mean age was 36 years, with most participants practicing running three times a week (55.4\%) and in the total sample, 43.2\% had an injury, with the knee (52.6\%) being the most commonly affected. Among those who had suffered injury, there was a strong correlation between the number of injuries and the time spent practicing this sport $(r=0.269)$ and those who trained in higher average daily distances $(r=0.226)$. However, the data showed that preventive work was not effective in reducing the incidence of lesions ( $r=-0.133)$. Conclusion: There is a direct relationship between the distance run and injury, as well as the time spent practicing the sport. Professional guidance does not have a significant influence in reducing injuries, i.e., many injuries may be due to the volume and intensity of training that is incorrectly prescribed or executed, not respecting the current physical condition and the biological individuality, targeting only the perspective to overcome the athlete's own limitations at any cost. We point out the importance of more studies that associate the progression of training, injuries, and prevention.

Keywords: running; wounds and injuries; physical education and training.

\section{RESUMEN}

Introducción: Hubo un marcado aumento en la popularidad de las carreras de calle; en consecuencia, hay más seguidores de esta práctica. De este modo, varios estudios han buscado diferentes enfoques de la modalidad con el objetivo de desvendar los mecanismos que lo componen. Objetivo: Verificar la incidencia de lesiones en practicantes de carrera de calle del municipio de Criciúma, SC, así como otros factores relacionados, tales como el perfil de los practicantes, las características del entrenamiento, y la existencia de un acompañamiento profesional. Métodos: Se realizó un estudio descriptivo transversal de 88 corredores (56 hombres y 32 mujeres). Resultados: La edad promedio fue de 36 años, con la mayoría de los participantes practicando carrera tres veces a la semana $(55,4 \%)$ y de la muestra total, el 43,2\% tenían una lesión, siendo la rodilla $(52,6 \%)$ más comúnmente afectada. Entre los que habían sufrido lesiones, hubo una fuerte correlación entre el número de lesiones y el de tiempo de práctica $(r=0,269)$, y los que recorrían 
mayor distancia promedio diaria $(r=0,226)$. Sin embargo, los datos mostraron que el trabajo preventivo no fue efectivo en la reducción de la incidencia de las lesiones $(r=-0,133)$. Conclusión: Existe una relación directa entre la distancia recorrida y lesiones, así como el tiempo de práctica. La orientación profesional no tiene influencia significativa en la reducción de las lesiones, es decir, muchas lesiones pueden deberse al volumen y la intensidad de entrenamiento que se prescribe o que no es correctamente realizado, sin respetar la condición física actual y la individualidad biológica con el fin de superar sus propios límites de todas las maneras. Enfatizamos la importancia de realizar más estudios que relacionan la progresión del entrenamiento, las lesiones y la prevención.

Descriptores: carrera; heridas y traumatismos; educación y entrenamiento físico.

\section{INTRODUÇÃO}

A prática regular e sistemática de atividade física tem sido motivo de intensas pesquisas na perspectiva de cada vez mais demonstrar os benefícios deste hábito. Do ponto de vista de prevenção a doenças crônicas, as duas maiores instituições de estudo em saúde e exercício físico, o American College of Sports Medicine (ACSM) e a American Heart Association (AHA) preconizam a prática de atividades físicas de longa duração, intensidade moderada, que envolvam grandes grupamentos musculares, ou seja, exercícios preponderantemente aeróbicos, pois apresentam poucas restrições ${ }^{1}$. Estas características podem ser relacionadas com a modalidade de corrida² ${ }^{2}$ que atualmente contempla grande número de praticantes, devido ao baixo custo e os benefícios que apresentam ${ }^{3}$, como melhora na sensibilidade a insulina, redução de quantidades de gordura corporal e concentrações de triglicerídeos, LDL e colesterol total, aumentos de massa magra e óssea, potência aeróbica e capacidade antioxidante, redução da pressão arterial pós-exercício, ou seja, todos fatores importantes e relevantes na melhora da qualidade de vida 4 .

No entanto, recentes estudos sobre corrida têm demonstrado forte correlação com incidência de lesões quando relacionado a fatores intrínsecos ${ }^{5}$ como: anormalidades biomecânicas e anatômicas, menor flexibilidade, histórico de lesões, características antropométricas, densidade óssea, composição corporal ${ }^{6,7}$ e maior força muscular ${ }^{8}$, e extrínsecos ligados a preparação ou prática da corrida como: duração da sessão e longa quilometragem semanal ${ }^{9}$, erros de planejamento e execução do treinamento, tipo de superfície de treino, tipo de percurso, tipo de calçado, alimentação, hidratação e prática concomitante de outras modalidades esportivas entre outros possíveis fatores, mas não citados neste trabalho.

Tonoli et al. ${ }^{10}$ descrevem que corredores iniciantes são os mais propensos a lesões, em alguns casos pelo fato de não terem certa experiência na modalidade. Em corredores de longa distância recreativos elas estão relacionadas à frequência semanal de corrida baixa. Em competidores de longa distância a maior incidência de lesões foi encontrada nos praticantes de seis ou mais vezes semanalmente e nos que não utilizavam calçados ortopédicos adequados. Nos maratonistas os fatores significantes foram o histórico de lesões e a idade, já nos corredores de cross country as lesões estão relacionadas com o ângulo do quadríceps nas subidas inerentes aos percursos.

Relacionado à incidência de lesões, observa-se uma variação entre 19,4\% e 92,4\% em membros inferiores, sendo o joelho a região mais acometida ${ }^{11}$. Hino et al. ${ }^{12}$ verificou que $1 / 3$ do sexo masculino e $1 / 4$ do sexo feminino sofreram alguma lesão nos seis meses que antecederam o início das provas de corrida de rua. Ferreira et al. ${ }^{13}$ verificou uma prevalência de lesões entre 40\% em 100 atletas amadores, já Pazin et al. ${ }^{9}$ identificou uma prevalência de 37,7\% de lesões em 115 participantes de provas de corrida de rua.

Desta forma a prática de corrida de rua requer cuidados essenciais, e, tanto o profissional da área quanto o praticante devem ter conhecimentos sobre as variáveis desta prática minimizando assim as possíveis consequências. Portanto o objetivo do presente estudo foi avaliar a incidência de lesões em corredores de rua do município de Criciúma, SC, Brasil, determinando o perfil dos corredores, as principais lesões, os locais de corrida dos participantes, o controle de cargas e outras variáveis do treinamento, se há acompanhamento profissional, na perspectiva de correlacionar as características do treinamento com a incidência de lesões.

\section{MÉTODOS}

A pesquisa caracteriza-se como descritiva, transversal, tendo sido realizada por meio de um questionário. Este estudo foi aprovado pelo Comitê de Ética da Universidade do Extremo Sul Catarinense (UNESC), Brasil, sob o protocolo 419.022.

A população foi constituída por corredores de ambos os sexos, com idade entre 18 a 70 anos, com tempo de prática igual ou maior que três meses, com frequência semanal de no mínimo duas vezes na semana, com ou sem orientação e tempo superior a 20 min por treino. A amostra foi constituída por 88 corredores voluntários (56 do sexo masculino e 32 do sexo feminino) que atenderam a todos os critérios de inclusão e foi representativa ao objetivo do estudo.

Como critérios de inclusão foram estabelecidos: idade acima de 18 anos, ter no mínimo experiência de três meses de prática, com frequência semanal de no mínimo duas vezes, tempo mínimo por sessão de 20 min, devolver os instrumentos de investigação preenchidos e no prazo, assinar e devolver o termo de consentimento livre esclarecido.

Como critério de exclusão, o não preenchimento de qualquer dos itens de inclusão, além de apresentar problemas de saúde e limitações físicas.

Primeiramente foi elaborado um questionário buscando atender os objetivos desta pesquisa, contendo perguntas abertas e fechadas, para validação das questões propostas foi realizada a análise de conteúdo pelos docentes da Instituição. Posteriormente o pesquisador entrou em contato com as empresas do ramo fitness e profissionais que oferecem o serviço de corrida individual ou em grupo, apresentando o projeto e solicitando a autorização para aplicar o instrumento aos seus clientes. Para os adeptos a corrida que praticam sem esse tipo de orientação, a coleta dos dados foi realizada nos locais: Parque das Nações Cincinato Naspolini, Paço Municipal e Arredores do Colégio Marista Criciúma, onde os corredores eram abordados durante seu treino e respondiam o questionário após o término do mesmo para o pesquisador responsável. Houveram apenas três casos em que os questionários foram respondidos via e-mail, com uma autorização redigida no próprio termo de Consentimento Livre e Esclarecido. Posterior à coleta, os dados foram categorizados e organizados para a elaboração das discussões e conclusões do estudo. Ressaltamos que todos os dados utilizados foram do conhecimento do pesquisador, inclusive o peso e estatura dos sujeitos.

A pesquisa não apresenta risco algum para os entrevistados, em contraponto os dados aqui obtidos poderão contribuir para uma análise e consequentemente possíveis mecanismos de prevenção para as lesões identificadas e continuidade no treinamento. 
Os dados obtidos com essa pesquisa têm objetivos estritamente científicos e acadêmicos para divulgação em eventos científicos. Todos os dados estão seguros em um microcomputador protegido por senha com acesso restrito, de propriedade do pesquisador.

\section{Análise dos dados}

Os dados foram tabulados na planilha eletrônica Excel do Microsoft Office 2010 (Santa Rosa, Califórnia) e categorizados de acordo com o questionário. Posteriormente foram estratificados e analisados pelo pacote estatístico Statistical Package for the Social Sciences (SPSS) for Windows 20.0 (IMB, EUA), com nível de significância de $p<0,05$. Para os dados descritivos foi utilizada frequência absoluta, relativa, média e desvio padrão, o teste de normalidade foi o de Poison, a comparação de médias o teste $t$ de Student para amostras independentes e o teste de Spearman's para a correlação dos dados coletados.

\section{RESULTADOS}

Após uma revisão de literatura foi possível observar uma série de fatores referentes à incidência de lesões em corredores de rua, como: fatores de risco, localização de lesões, relação entre nível de condição física do corredor e lesões. A maior parte dos indivíduos que participaram do estudo (31,8\%) encontram-se na faixa etária de 31 a 40 anos de idade, representando uma média de 37,4 anos para os homens e 33,7 para as mulheres. Com relação ao IMC (índice de massa corporal), a maioria dos participantes do sexo masculino (64\%) apresentou-se com sobrepeso, enquanto no sexo feminino quase todas (91\%) estão em seu peso normal. Sobre o tempo de prática da modalidade de corrida, houve maior proporção da amostra que relatou correr há mais de cinco anos, entretanto, isso ocorreu com o sexo masculino (32\%), já que no feminino, em sua maioria (34\%), corriam há um período entre 1 e 2 anos, ambos os sexos treinando no período noturno (75\%) (Tabela 1). Com relação ao local de treino, grande parte dos participantes evitam correr em um único local $(51,2 \%)$ e o mais citado $(55,7 \%)$ foi o Parque das Nações Cincinato Naspolini.

Na Tabela 2 estão representados os dados relacionados às variáveis de treinamento analisadas relacionando-as com o sexo. Identificou-se que a frequência semanal de treino de três vezes tem maior incidência (53,5\%), assim como treinos que duram 60 min (49\%). A distância média diária percorrida pelos participantes do sexo masculino mais citada (27\%) é de $10 \mathrm{~km}$, enquanto nas mulheres (25\%) é de $5 \mathrm{~km}$. Quanto à orientação específica nos treinos, 52\% da amostra possui alguma orientação, sendo do sexo feminino (62,5\%), das quais a maioria (55\%) são orientadas por profissional de educação física.

A Tabela 3 identifica os fatores referentes à incidência de lesões, observa-se que $56,9 \%$ dos corredores realizam algum trabalho que julgam preventivo, sendo musculação o mais citado tanto no sexo masculino $(55,2 \%)$ quanto no feminino $(34,4 \%)$ e quando perguntados sobre a prática de outro exercício físico o mais citado também foi a musculação (44,4\%). Quanto a lesões desde o início da prática de corrida 56,8\% descrevem que nunca tiveram lesão. Nos que apresentam lesão, a maior incidência foi nos joelhos $(52,6 \%)$ tanto nos homens $(42,3 \%)$ quanto nas mulheres (75\%). Quando referido na tabela "outras lesões", corresponde a lesões de lombar, inflamação na coxa, tendão calcâneo, nervo ciático, pés, lombar, pescoço e entorse de tornozelo. 70,5\% dos participantes não sofreram nenhuma lesão no último ano, e as mais incidentes foram nos joelhos nos corredores do sexo masculino (35,3\%), enquanto no feminino a maior parte $(44,4 \%)$ foi na coxa. Lesões de quadril, costas, tornozelo e pescoço estão classificadas em "outras lesões". Sobre o tratamento dessas lesões, dos corredores do sexo masculino que já se lesionaram alguma vez, 20 $(74,1 \%)$ o fizeram, sendo fisioterapia o mais realizado (55\%) e nas 12 (83,3\%) mulheres que já se lesionaram alguma vez e realizaram algum tratamento, $50 \%$ fizeram reforço muscular.
Tabela 1. Descrição de variáveis de perfil dos participantes, subdivididos por gênero.

\begin{tabular}{|c|c|c|c|c|c|c|c|c|}
\hline & & \multicolumn{6}{|c|}{ Sexo } & \multirow{3}{*}{ Total } \\
\hline \multirow{2}{*}{\multicolumn{2}{|c|}{ Variáveis de perfil }} & \multicolumn{3}{|c|}{ Masculino } & \multicolumn{3}{|c|}{ Feminino } & \\
\hline & & f & $\%$ & $\bar{x} \pm D P$ & $f$ & $\%$ & $\bar{x} \pm D P$ & \\
\hline \multirow{5}{*}{$\begin{array}{l}\text { Idade dos } \\
\text { corredores }\end{array}$} & até 20 anos & 1 & 2 & \multirow{5}{*}{$\begin{array}{c}37,4 \pm \\
10,7\end{array}$} & 1 & 2 & \multirow{5}{*}{$\begin{array}{c}33,7 \pm \\
8,8\end{array}$} & 2 \\
\hline & 21 a 30 anos & 16 & 29 & & 12 & 21 & & 28 \\
\hline & 31 a 40 anos & 18 & 32 & & 10 & 18 & & 28 \\
\hline & 41 a 50 anos & 13 & 23 & & 9 & 16 & & 22 \\
\hline & Mais de 51 anos & 8 & 14 & & 0 & 0 & & 8 \\
\hline \multirow{3}{*}{ IMC } & Peso normal & 18 & 32 & \multirow{3}{*}{$\begin{array}{c}25,7 \pm \\
2,3\end{array}$} & 29 & 91 & \multirow{3}{*}{$\begin{array}{c}22,2 \pm \\
2,1\end{array}$} & 46 \\
\hline & Sobrepeso & 36 & 64 & & 3 & 9 & & 39 \\
\hline & Obesidade grau I & 2 & 4 & & 0 & 0 & & 2 \\
\hline \multirow{7}{*}{$\begin{array}{c}\text { Tempo de } \\
\text { prática }\end{array}$} & 0 a 6 meses & 5 & 9 & \multirow{7}{*}{$\begin{array}{l}71 \pm \\
89,3\end{array}$} & 2 & 6 & \multirow{7}{*}{$\begin{array}{l}46 \pm \\
78,9\end{array}$} & 7 \\
\hline & 7 a 12 meses & 10 & 18 & & 8 & 25 & & 18 \\
\hline & 13 a 24 meses & 4 & 7 & & 11 & 34 & & 15 \\
\hline & 25 a 36 meses & 9 & 16 & & 3 & 9 & & 12 \\
\hline & 37 a 48 meses & 6 & 11 & & 3 & 9 & & 9 \\
\hline & 49 a 60 meses & 4 & 7 & & 2 & 6 & & 6 \\
\hline & 61 a 430 meses & 18 & 32 & & 3 & 9 & & 21 \\
\hline \multirow{4}{*}{$\begin{array}{c}\text { Horário } \\
\text { habitual do } \\
\text { treino }\end{array}$} & Matutino & 4 & 7 & & 4 & 13 & & 8 \\
\hline & Vespertino & 6 & 11 & & 4 & 13 & & 10 \\
\hline & Noturno & 43 & 77 & & 23 & 72 & & 66 \\
\hline & $\begin{array}{c}\text { Períodos } \\
\text { alternados }\end{array}$ & 3 & 5 & & 1 & 3 & & 4 \\
\hline \multicolumn{2}{|c|}{ Total } & \multicolumn{3}{|c|}{56} & \multicolumn{3}{|c|}{32} & 88 \\
\hline
\end{tabular}

IMC: Índice de Massa Corporal; f: Frequência absoluta; \%: Frequência relativa; : Média; DP: Desvio Padrão.

Tabela 2. Descrição das variáveis de treinamento identificadas, subdivididos por gênero.

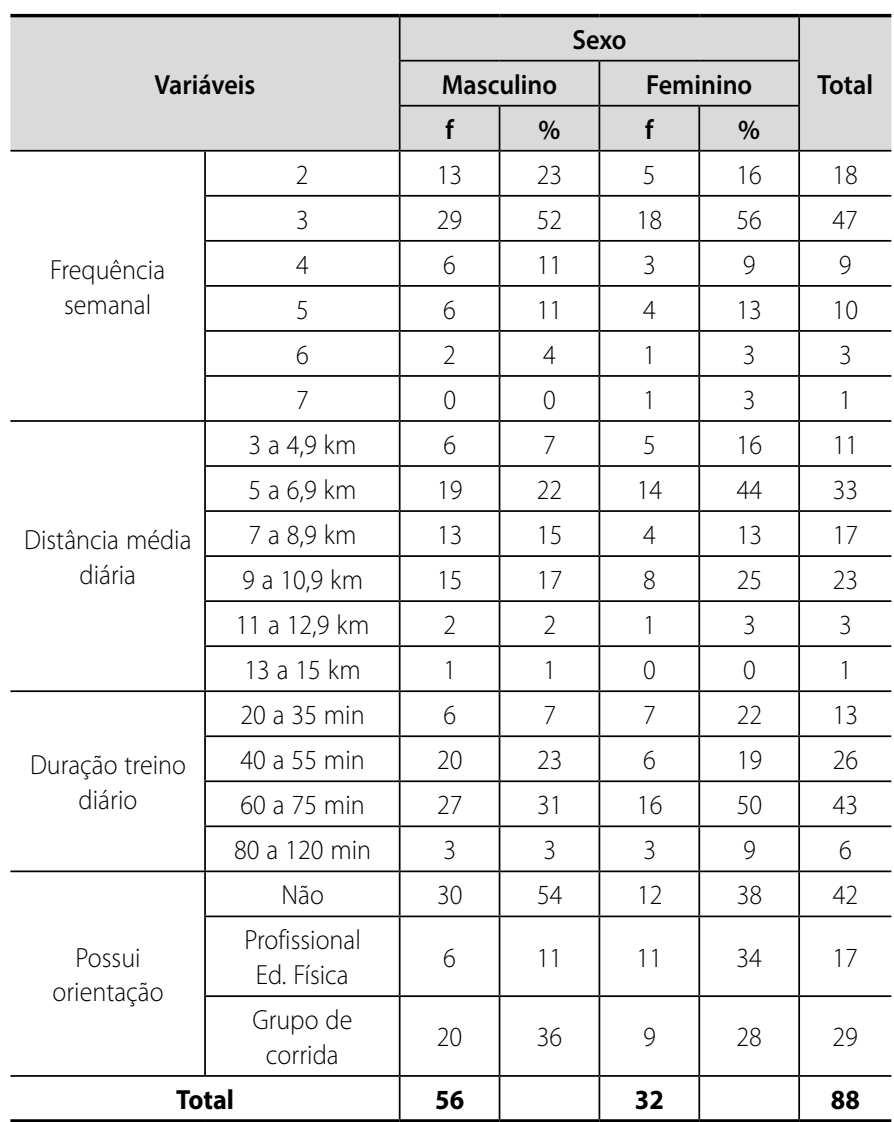

f: Frequência absoluta; \%: Frequência relativa. 
A Tabela 4 relata dados sobre queixas de dores atuais nos corredores, dos quais vinte e quatro $(27,3 \%)$ têm na região do joelho, vinte e oito $(31,8 \%)$ na região lombar, dezesseis $(18,2 \%)$ no pescoço, nove $(10,2 \%)$ na região do quadril, dez $(11,4 \%)$ nos pés ou tornozelos e treze $(14,8 \%)$ na região das pernas, estas três últimas representadas na tabela como "outras dores". Na descrição do período de persistência das dores, a maioria relatou que essas dores não persistiam por períodos maiores que sete dias.

Por fim correlacionou-se alguns dados de perfil e características de treinamento com a incidência de lesões (Tabela 5), demonstrando uma correlação positiva entre quantidade de lesões e o tempo de prática da modalidade, logo, quanto mais tempo os corredores praticam a modalidade maior é a quantidade de lesões que eles estão sujeitos. Observou-se também que a maior distância média diária de treino aumenta o risco

Tabela 3. Descrição das variáveis de lesão e prevenção, subdivididos por gênero.

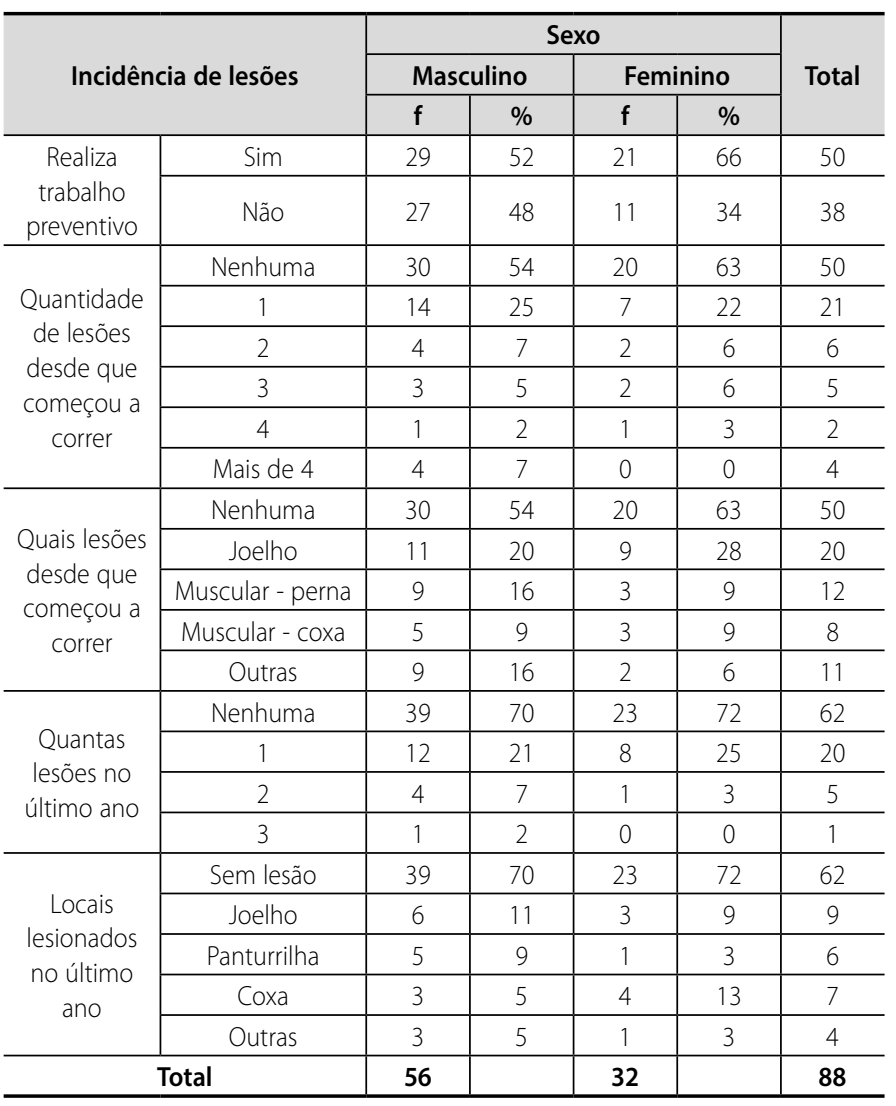

f:Frequência absoluta; \%: Frequência relativa. de lesões, da mesma forma que uma maior idade pode refletir em um maior número de lesões, no entanto, ao contrário do que se esperava, os dados demonstram que realizar trabalho preventivo não tem interferido diretamente numa menor quantidade de lesões.

\section{DISCUSSÃO}

É comum observarmos estudos identificando o perfil dos praticantes de determinadas atividades físicas. Um dos objetivos específicos desse estudo foi verificar o perfil dos praticantes de corrida de rua de Criciúma, SC e, comparando a outros estudos, foi possível observar algumas semelhanças interessantes, por exemplo, a média de idade foi de 36 anos, valor muito próximo ao estudo de Ferreira et al. ${ }^{13} \mathrm{com}$ uma média de idade de 34,7 anos. Com relação à orientação especializada, em que este estudo encontrou uma frequência de $46 \%$ dos que a possuíam, Pazin et al. ${ }^{9}$ verificou em sua pesquisa que $40,9 \%$ dos seus entrevistados tinham orientação. Além destes houve dados próximos quanto ao horário de treino, que na presente pesquisa o mais citado foi o noturno (75\%), assim como nos corredores de Belo Horizonte ${ }^{13}$ (53\%).

É de fundamental importância tanto aos profissionais que prescrevem a prática de corrida quanto aos corredores desvendarem cada vez mais a modalidade, buscando aperfeiçoar o rendimento e minimizar os riscos para possíveis lesões, tendo em vista que muitos estudos apontam considerável incidência de lesões em corredores de rua, assim como seus fatores de risco. Em estudos nacionais ${ }^{9,12,13}$ houve uma variação entre 25\% e 40\% na incidência de lesões enquanto em estudos internacionais Van Gent et al. ${ }^{11}$ identificou essa variação entre 19,4\% e 92,4\% em lesões de membros inferiores. O presente estudo, cuja análise envolveu 88 corredores, verificou a incidência de 43,2\%, no entanto não houve apenas lesões de membros inferiores e o questionamento sobre as mesmas era referente a todo tempo de corrida do praticante, não apenas nos últimos seis meses como em estudo de Hino et al. ${ }^{12}$, logo, há um indicativo de correlação entre o tempo de prática com lesão. Observando a elevada incidência de lesões surge a necessidade de desenvolver medidas de prevenção levando em conta que o número de adeptos a prática de corrida de rua cresce a cada ano ${ }^{3}$.

Um dos dados de difícil análise foi relacionado à prevenção, com uma grande divergência nos resultados talvez pela compreensão de o que são atividades preventivas, ou seja, alguns acreditam que o alongamento, a musculação, o Pilates ou o treinamento funcional tenham o papel em seus programas de treinamento como trabalho preventivo, outros, porém, compreendem como outras modalidades e não os consideram como preventivos. Correlacionando dados de trabalho preventivo e

Tabela 4. Dados sobre queixas de dor atualmente, subdivididos por gênero.

\begin{tabular}{|c|c|c|c|c|c|c|c|c|c|c|c|c|c|c|c|c|}
\hline \multirow{3}{*}{ Dor atualmente } & \multicolumn{4}{|c|}{ Região dos joelhos } & \multicolumn{4}{|c|}{ Região lombar } & \multicolumn{4}{|c|}{ Pescoço } & \multicolumn{4}{|c|}{ Outras } \\
\hline & \multicolumn{2}{|c|}{ Sim } & \multicolumn{2}{|c|}{ Não } & \multicolumn{2}{|c|}{ Sim } & \multicolumn{2}{|c|}{ Não } & \multicolumn{2}{|c|}{ Sim } & \multicolumn{2}{|c|}{ Não } & \multicolumn{2}{|c|}{ Sim } & \multicolumn{2}{|c|}{ Não } \\
\hline & f & $\%$ & f & $\%$ & f & $\%$ & f & $\%$ & f & $\%$ & f & $\%$ & f & $\%$ & f & $\%$ \\
\hline Masculino $(\mathrm{N}=56)$ & 16 & 29 & 40 & 71 & 21 & 38 & 35 & 63 & 10 & 18 & 46 & 82 & 20 & 36 & 36 & 64 \\
\hline Feminino $(\mathrm{N}=32)$ & 8 & 25 & 24 & 75 & 7 & 22 & 25 & 78 & 6 & 19 & 26 & 81 & 12 & 38 & 20 & 63 \\
\hline Total & 24 & 27,3 & 64 & 72,7 & 28 & 31,8 & 60 & 68,2 & 16 & 18,2 & 72 & 81,8 & 9 & 10,2 & 79 & 89,8 \\
\hline
\end{tabular}

N:Total de participantes; $f$ : Frequência absoluta; $\%=$ Frequência relativa.

Tabela 5. Valores de correlação entre variáveis.

\begin{tabular}{|c|c|c|c|c|c|c|c|c|c|c|c|c|c|}
\hline \multicolumn{3}{|c|}{ Correlação } & $\mathrm{N}$ & TP & 1 & $\mathbf{P}$ & FS & DM & DT & 0 & PR & FE & IMC \\
\hline \multirow{4}{*}{ Spearman's rho } & \multirow{2}{*}{$\begin{array}{c}\text { Quantidade de lesões desde } \\
\text { que começou a correr }\end{array}$} & $r$ & \multirow{2}{*}{88} & $0,269^{*}$ & 0,189 & 0,096 & 0,106 & $0,226^{*}$ & 0,114 & 0,137 & $-0,133$ & 0,010 & 0,060 \\
\hline & & Sig. (2-tailed) & & 0,011 & 0,078 & 0,376 & 0,325 & 0,034 & 0,288 & 0,202 & 0,216 & 0,929 & 0,580 \\
\hline & \multirow{2}{*}{$\begin{array}{c}\text { Quantidade de lesões no } \\
\text { último ano }\end{array}$} & $r$ & \multirow{2}{*}{88} & 0,077 & 0,080 & 0,082 & 0,034 & 0,093 & 0,072 & 0,123 & $-0,116$ & $-0,028$ & 0,039 \\
\hline & & Sig. (2-tailed) & & 0,476 & 0,457 & 0,450 & 0,750 & 0,387 & 0,503 & 0,254 & 0,282 & 0,798 & 0,722 \\
\hline
\end{tabular}

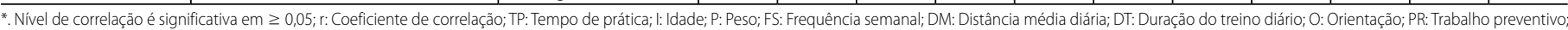
FE: Frequência semanal outro exercício; IMC: Índice de Massa Corporal. 
quantidade de lesões chama a atenção que realizá-lo não tem interferido diretamente numa menor incidência de lesões.

Durante a elaboração do instrumento de coleta, foi necessário estabelecer um critério específico para definição de lesão, então foi adotado o de Lun et al. ${ }^{5}$, que a descreve como qualquer dor ou agravo que tenha limitado ou afastado o atleta de treinos ou competições por um ou mais dias. Entende-se que esta não seja capaz de estabelecer alguma gravidade a lesão, no entanto com ela é possível identificar fatores como localização e natureza da mesma. Os dados deste estudo são corroborados com outros estudos revisados por Wen ${ }^{6}$ eTonoli et al. ${ }^{10}$, que também verificaram uma maior incidência de lesões nos joelhos. Várias são as investigações sobre o porquê dessa localização, no entanto são muitos os fatores que podem colaborar para o surgimento dessas lesões. Há algum tempo, com o frequente avanço das tecnologias, vêm se descartando a ideia de que os corredores se lesionem por utilizar calçado inadequado e reforçando a ideia de que as variáveis do treinamento ${ }^{14}$ como intensidade ${ }^{15}$ e volume $e^{5,16,17}$ sejam as principais causas, assim como neste estudo, que verificou correlação positiva $(r=0,226)$ entre incidência de lesões e distância média diária, conforme demonstra a Tabela 5. Sobre as variáveis intrínsecas ${ }^{5}$ esta pesquisa não encontrou correlações significativas entre lesão e IMC ou peso, no entanto, conforme mostra a Tabela 1, grande parte (64\%) dos corredores do sexo masculino estão com sobrepeso, fator que pode ser o grande influenciador no ingresso de homens à prática da modalidade ou reflete que, já que muitos $(44,4 \%)$ praticam a musculação, a informação de IMC possa estar mascarando o alto percentual de massa magra, porém o estudo não teve entre seus objetivos responder a este questionamento. Outro valor preocupante obtido é a quantidade de praticantes que treinam e persistem em sentir alguma dor, que foram na maioria queixas na região lombar no sexo masculino (38\%) e na região do joelho no sexo feminino (25\%).

Verifica-se então que estudos recentes demonstram uma grande incidência de lesões em corredores de rua, fato que aparentemente não tem interferido negativamente no crescimento do número de praticantes, porém é muito negativo ter tantos lesionados presentes na população, já que a prática física para essa parcela deveria significar promoção de saúde e um estilo de vida mais saudável ${ }^{12}$.

As principais limitações deste estudo são comuns em pesquisas que buscam identificar incidência de lesões em atividades físicas, que são as divergências quanto ao entendimento de lesão e prevenção, suas causas e ao possível esquecimento de lesões nos praticantes há muito tempo.

\section{CONCLUSÃO}

Conclui-se que dentre os fatores relacionados a lesão destacam-se a grande quantidade de já lesionados (43,2\%) e o joelho como localização mais frequente $(52,6 \%)$ percebendo haver uma relação direta entre distância percorrida e lesão, bem como o tempo de prática. A orientação de profissionais não apresenta influência significativa na redução das lesões, ou seja, muitas lesões podem ser decorrentes de volumes e intensidade de treinos prescritos ou executados de maneira equivocada, não respeitando condicionamento físico atual e a individualidade biológica, na perspectiva de superar seus próprios limites de qualquer modo. Ressalta-se a importância de mais estudos que relacionem a progressão de treinamento, lesão e prevenção.

Todos os autores declararam não haver qualquer potencial conflito de interesses referente a este artigo.

CONTRIBUIÇÕES DOS AUTORES: Cada autor contribuiu individual e significativamente para o desenvolvimento do manuscrito. GMMR (0000-0002-6333-4812)* foi responsável pela escrita inicial, coleta e discussão dos dados. JMF (0000-0003-2843-6482)* contribuiu na construção do projeto, correções gerais, analise dos dados e fechamento do artigo. Ambos os autores contribuíram com o conceito intelectual do estudo.*ORCID (Open Researcher and Contributor ID).

\section{REFERÊNCIAS}

1. Pileggi P, Gualano B, Souza M, Caparbo VF, Pereira RMR, Pinto ALS, et al. Incidência e fatores de risco de lesões osteomioarticulares em corredores: um estudo de coorte prospectivo. Rev. bras. Educ. Fís. Esporte. 2010;24(4):453-62.

2. Pedersen BK, Saltin B. Evidence for prescribing exercise as therapy in chronic disease. Scand J Med Sci Sports. 2006;16(Suppl 1):3-63.

3. Salgado JVV, Chacon-Mikahil MPT. Corrida de rua: análise do crescimento do número de provas e de praticantes. Conexöes. 2006;4(1):90-9.

4. Paluska SA. An overview of hip injuries in running. Sports Med 2005;35(11):991-1014.

5. Lun V, Meeuwisse WH, Stergiou P, Stefanyshyn D. Relation between running injury and static lower limb alignment in recreational runners. Br J Sports Med. 2004;38(5):576-80.

6. Wen DY. Risk factors for overuse injuries in runners. Curr Sports Med Rep. 2007;6(5):307-13.

7. Gellman R, Burns S. Walking aches and running pains. Injuries of the foot and ankle. Prim Care. 1996;23(2):263-80.

8. Messier SP, Legault C, Schoenlank CR, Newman JJ, Martin DF, DeVita P. Risk factors and mechanisms of knee injury runners. Med Sci Sports Exerc. 2008;40(11):1873-9.

9. Pazin J, Duarte MFS, Poeta LS, Gomes MA. Corredores de rua: características demográficas, treinamento e prevalência de lesões. Rev Bras Cineantropom Desempenho Hum. 2008;10(3)3:277-82.
10. Tonoli C, Cumps E, Aerts I, Verhagen E, Meeusen R. Incidence, risk factors and prevention of running related injuries in long-distance runners: a systematic review. Sport \& Geneeskunde. 2010;5:12-18.

11. van Gent RN, Siem D, van Middelkoop M, van Os AG, Bierma-Zeinstra SM, Koes BW. Incidence and determinants of lower extremity running injuries in long distance runners: a systematic review. $\mathrm{Br} J$ Sports Med. 2007;41(8):469-80.

12. Hino AAF, Reis RS, Rodriguez-Anez CR, Fermino RC. Prevalência de lesões em corredores de rua e fatores associados. Rev Bras Med Esporte. 2009;15(1):36-9.

13. Ferreira AC, Dias JMC, Fernandes RM, Sabino GS, Anjos MTS, Felício DC. Prevalência e fatores associados a lesões em corredores amadores de rua do município de Belo Horizonte, MG. Rev Bras Med Esporte. 2012;18(4):252-5

14. Hreljac A, Marshall RN, Hume PA. Evaluation of lower extremity overuse injury potential in runners. Med Sci Sports Exerc. 2000;32(9):1635-41.

15. Sallade JR, Koch S. Training errors in long distance running. J AthI Train. 1992;27(1):50-3.

16. Hootman JM, Macera CA, Ainsworth BE, Martin M, Addy CL, Blair SN. Association among physical activity level, cardiorespiratory fitness, and risk of musculoskeletal injury. Am J Epidemiol. 2001;154(3):251-8.

17. Fredericson M, Misra AK. Epidemiology and aetiology of marathon running injuries. Sports Med. 2007;37(4-5):437-9 